\title{
A method for hybrid fire testing: development, implementation and numerical application
}

\author{
A. Sauca, E. Mergny, T. Gernay \& J.M. Franssen \\ University of Liege, Liege, Belgium
}

\begin{abstract}
Hybrid Fire Testing (HFT) is a technique that allows assessing experimentally the fire performance of a structural element under real boundary conditions that capture the effect of the surrounding structure. To enable HFT, there is a need for a method that is unconditionally stable, ensures equilibrium and compatibility at the interface and captures the global behaviour of the analysed structure. A few attempts at conducting HFT have been described in the literature, but it can be shown, based on the analytical study of a simple one degree-of-freedom elastic system, that the considered method was fundamentally unstable in certain configurations which depend on the relative stiffness between the two substructures, but which cannot be easily predicted in advance. In this paper, a new method is introduced to overcome the stability problem and it is shown through analytical developments and applicative examples that the stability of the new method does not depend on the stiffness ratio between the two substructures. The new method is applied in a virtual hybrid test on a $2 \mathrm{D}$ reinforced concrete beam part of a moment resisting frame, showing that stability, equilibrium and compatibility are ensured on the considered multiple degree-of-freedom system. Besides, the virtual HFT succeeds in reproducing the global behaviour of the analysed structure. The method development and implementation in a virtual (numerical) setting is described, the next step being its implementation in a real (laboratory) hybrid test.
\end{abstract}

\section{INTRODUCTION}

New materials or structural components require performing fire tests to understand the behavior of these elements when exposed to elevated temperatures. The most common practice is to perform standard tests on individual structural elements, neglecting the effect of the surrounding. During these tests, the mechanical or kinematic boundary conditions are fixed (i.e. constant through time). Hybrid fire testing represents a promising technique for keeping the advantage of testing only parts of the structure while at the same time considering the global behavior of the structure. In a hybrid test, a single element is physically tested but the boundary conditions are updated during the test to model the effect of the surrounding on the tested element.

Hybrid fire testing is not widely explored compared to hybrid seismic testing, where this technique has been first applied in the early 1970s. Yet, although it has been extensively described in other fields, Saouma and Sivaselvan (2008), the straightforward application from one field to another is not possible. The main challenge in hybrid fire tests is related to the necessity of conducting the tests in real time since the temperature distribution in most elements is highly nonuniform and time dependent, except for metallic elements in which a uniform temperature distribution can develop and the test can be performed in the temperature domain (assuming that creep is not significant). Moreover, the interface boundary conditions are changing continuously under the effect of thermal expansion. The key component of HFT is the physical substructure (PS) which is tested in a furnace. The PS is physically tested because the behavior in the fire situation is unknown or cannot be simulated. Another component of HFT is the numerical substructure 
(NS) the response of which is analyzed aside during the test in a finite element model or by a predetermined matrix. The NS refers to the remainder of the structure and the response of this structure will have an influence on the boundary conditions of the tested element.

Few attempts at HFT have been done in the past. The first documented attempt to perform HFT was made by Korzen et al. (2002) at BAM (Germany) on a column specimen extracted from a building. Only the axial degree-of-freedom is controlled during the test and the NS is defined by a constant matrix. Robert et al. (2010) at CERIB (France) presents a HFT performed on a concrete slab where the behavior of the NS is modeled by a predetermined matrix. Three degrees-of-freedom are controlled, namely the axial elongation and the rotations on the two supports. Mostafaei (2013a, 2013b) at NRC (Canada) performed a HFT on a concrete column while the remainder structure, i.e. a moment resisting frame with some parts frame exposed to fire, was modeled in the nonlinear finite element software SAFIR ${ }^{\circledR}$ (Franssen, 2005). More recently, researchers worked on the development of the HFT methodology and validation has been done in the numerical environment (Tondini et al., 2016) or by experiments performed on small-scale specimens, i.e. Whyte et al. (2016) and Schulthess et al. (2016). In most cases, the methodology applied in the former HFT has been tailored for the analyzed case studies and for the capability of the fire facility where the test has been performed. The research objective is here to develop a methodology which is applicable independently on the type of the case study and the capability of the furnace. In this paper, the methodology considered in the former HFT performed on real structural elements, i.e. Korzen, Robert and Mostafaei, will be analyzed in details. Moreover, for a better understanding, the methodology considered in these three hybrid fire tests will be referred as the "first generation method". It will be shown that the considered methodology is applicable only for specific cases and a new solution will be proposed in order to perform HFT in a general context, independently on the analyzed case study. The capability of the proposed method will be analyzed on a case study consisting of a concrete beam extracted from a moment resisting frame.

\section{INTEREST OF HYBRID FIRE TESTING}

In order to highlight the potential of HFT, the behavior of a concrete beam extracted from a moment resisting frame will be analyzed numerically in different configurations (details about the analyzed moment resisting frame and the fire load can be found in Section 5).

The configurations considered are: (1) a test of the whole structure, only possible in a virtual environment (2) a test on the beam simply supported with no bending moment introduced at the supports (3) a test on the beam simply supported with constant bending moments introduced at the supports (through cantilever extensions, for example), (4) test on the beam with rotations fixed on the supports and free thermal expansion, (5) test on the beam under fixed rotations and fixed thermal expansions and (6) a hybrid fire test. For all these cases, the evolution of the mid-span displacements as a function of time is presented in Figure 1.

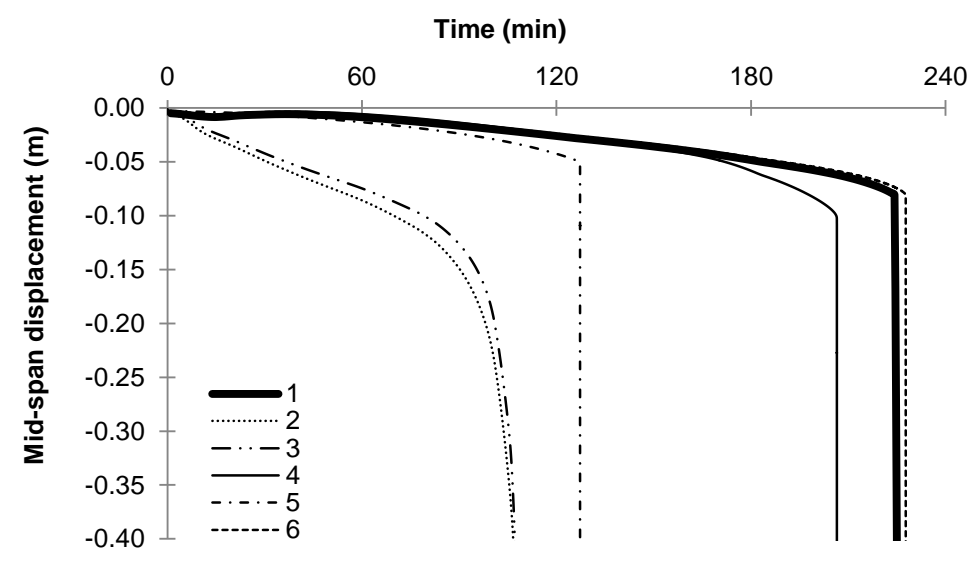

Figure 1. Mid-span displacement of the beam in different testing configurations 
The results of the test of the whole structure are considered as the reference. The failure time and the behavior of the structural element varies significantly in most configurations. From all these cases, the results of a full scale test (the global behavior) is approached only when performing HFT.

\section{THE "FIRST GENERATION METHOD"}

The first generation method is analyzed in this section considering a 1 degree-of-freedom linear system presented in Figure 2. The system is composed of two bars, the PS of length $L_{P}$ and the NS of length $L_{N}$. The heated PS is defined by the axial stiffness $K_{P}$ and thermal coefficient of the material $\alpha$ whereas the cold NS is characterized by the axial stiffness $K_{N}$. The temperature in the PS increases in time which induces thermal expansion of the single DoF, i.e. the axial displacement at node 2. For the sake of simplicity, the stiffness of the PS and NS remain constant. By an analytical analysis of the $1 \mathrm{DoF}$ linear system, it will be shown that the first generation method is conditionally stable depending on the stiffness ratio between the NS and PS. The stability conditionality is a consequence of the fact that during the HFT, only the characteristics of the NS are considered in the calculations, while the characteristics of the PS are neglected.

Hybrid fire tests can be performed in force control or displacement control procedure. In the physical tests, the displacement control procedure is usually preferred because it remains stable even in the last stage of the test. When stiff elements are tested, the force control procedure may be preferable, since the measured displacements can be infinitely small. In this case the errors in the measurements have an important impact on the reaction force and instability can occur. Therefore, the procedure to be applied should suit the type of the problem.

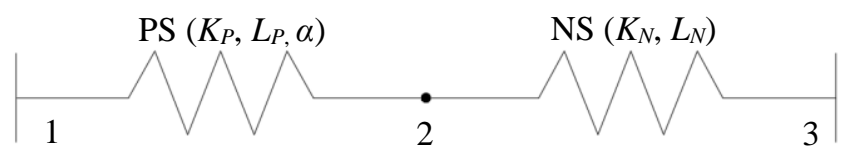

Figure 2. One degree-of-freedom linear system

To exemplify the hybrid fire procedure, the displacement control procedure is considered and applied step by step for the case study, i.e. 1 DoF linear system. The analytical expressions will be expressed for this simple situation.

a. First, the analysis of the entire system is performed in order to determine the forces and the displacements at the interface between the PS and NS before the fire starts. In this case, where no external force is applied, the displacement and force at the interface are null $\left(F_{P}\left(t_{0}\right)=F_{N}\left(t_{0}\right)=0\right.$ and $\left.u_{P}\left(t_{0}\right)=u_{N}\left(t_{0}\right)=0\right)$.

b. The PS is placed in the furnace (in a real HFT) and loaded with the exterior loads and interface conditions, while the NS is modeled aside. Herein the exterior loads, the interface forces and displacements are equal to zero.

c. Heating of the PS starts. In displacement control procedure, no thermal expansion of the PS is allowed (it is the displacement at the interface which is controlled and it remains fixed during one time step). The reaction force is measured. In this example, it yields to the value expressed by Equation 1.

$$
F_{P}\left(t_{1}\right)=-K_{P} \alpha L_{P} T\left(t_{1}\right)
$$

where:

$F_{x}\left(t_{n}\right)$ is the interface force of substructure $x$ (the subscript can be either P for the PS or N for NS) at time $t_{n}$ (i.e. force of node 2).

$T\left(t_{n}\right)$ is the temperature of the PS at time $t_{n}$.

$n$ is the number of the time step. 
d. The measured reaction force, i.e. Equation 1, is imposed on the NS. This generates a displacement that is computed using Equation 2.

$$
u_{N}\left(t_{1}\right)=\frac{K_{P}}{K_{N}} \alpha L_{P} T\left(t_{1}\right)
$$

where:

$u_{x}\left(t_{n}\right)$ is the interface displacement of substructure $x$ (P for the PS and N for NS) at time $t_{n}$ (i.e. displacement of node 2).

e. The new displacement is imposed on the PS (Equation 3). A time delay $\Delta t_{P}$ is used to capture the time needed to compute the reaction of the NS and to impose the displacement by the actuator, as for a real HFT.

$$
u_{P}\left(t_{1}+\Delta t_{P}\right)=\frac{K_{P}}{K_{N}} \alpha L_{P} T\left(t_{1}\right)
$$

f. The new displacement induces a new reaction force of the PS. Meanwhile, heating of the PS has continued and also induces variation in force. The updated reaction force of the PS at the interface $F_{P}\left(t_{2}\right)$ is measured (given here by Equation 4 ) and imposed on the NS. This generates a new displacement $u_{N}\left(t_{2}\right)$ given by Equation 5 .

$$
\begin{aligned}
& F_{P}\left(t_{2}\right)=-K_{P} \alpha L_{P}\left(T\left(t_{2}\right)-\frac{K_{P}}{K_{N}} T\left(t_{1}\right)\right) \\
& u_{N}\left(t_{2}\right)=\frac{1}{R} \alpha L_{P}\left(T\left(t_{2}\right)-\frac{K_{P}}{K_{N}} T\left(t_{1}\right)\right)
\end{aligned}
$$

Steps $e$ and $f$ are repeated during the entire hybrid fire test in order to maintain equilibrium and compatibility at the interface. For future discussion, the ratio between the stiffness of the NS and PS will be referred in this paper as stiffness ratio, $R=\frac{K_{N}}{K_{P}}$.

Expanding Equation 4 and 5, for $n$ time steps, the measured reaction force of the PS at any time step $t_{n}$ can be expressed by Equation 6 and the displacement generated by the NS by Equation 7 .

$$
\begin{aligned}
& F_{P}\left(t_{n}\right)=-K_{P} \alpha L_{P} \sum_{i=0}^{n-1}\left[\left(-\frac{1}{R}\right)^{i} T\left({ }_{n-i}\right)\right. \\
& u_{N}\left(t_{n}\right)=\frac{1}{R} \alpha L_{P} \sum_{i=0}^{n-1}\left[\left(-\frac{1}{R}\right)^{i} T\left({ }_{n-i}\right)\right]
\end{aligned}
$$

The same developments can be made for the force control procedure (Sauca et al. 2016a). In this case, the measured displacement at any time step $t_{n}$ can be determined using Equation 8 and the generated reaction force by the NS by Equation 9 .

$$
\begin{aligned}
& u_{P}\left(t_{n}\right)=\alpha L_{P} \sum_{i=0}^{n-1}\left[(-R)^{i} T\left(t_{n-i}\right)\right] \\
& F_{N}\left(t_{n}\right)=K_{N} \alpha L_{P} \sum_{i=0}^{n-1}\left[(-R)^{i} T\left(t_{n-i}\right)\right]
\end{aligned}
$$

From Equations 6 to 9 it is clear that the results during the HFT, using the first generation method, are influenced by the stiffness ratio $R$.

To avoid instability, the value in the parenthesis which involves the stiffness ratio should be smaller than 1, i.e. $R<1$, for the force control procedure and $R>1$ for displacement control procedure. If not, the computed value tends toward infinity when the number of iteration $i$ in- 
creases, irrespectively of the size of the time steps, and the process becomes unstable. A straightforward selection of the proper procedure based on the value of the stiffness ratio is nevertheless not possible because of the following reasons: (i) the stiffness ratio varies during the test; the fire exposure induces degradation of the stiffness of the PS, and the variation is not easy predictable before or during the HFT, and moreover, (ii) multiple DoFs controlled at the interface can require different procedures.

To avoid the instability which might occur prematurely during the hybrid fire tests, a novel method needs to be developed which can be applied independently of the stiffness ratio between the substructures.

\section{THE NEW PROPOSED METHOD}

The dependency on the stiffness ratio of the "first generation method" is due to the fact that the stiffness of the PS is neglected in the calculation of the solution; only the stiffness of the NS is accounted for. In the new method, the stiffness of the PS as well as that of the NS are taken into account.

The linear 1DoF system presented in the Section 3 is analyzed here with the new methodology. The analytical expressions will be presented for the case of the displacement control procedure.

Steps $a, b$ and $c$ are the same as in the case of the first generation method. The next steps are:

$\mathrm{d}$. The reaction forces of the NS are computed based on the stiffness of the NS sole using Equation 10. If the NS is heated, the reaction forces vary during one time step. If the NS is kept cold, then the reaction forces is constant during one time step.

$$
F_{N}\left(t_{1}\right)=K_{N} u\left(t_{0}\right)=0
$$

e. The measured reaction forces of the PS (Equation 1) are compared with the computed reaction forces of the NS. Generally, the equilibrium is not ensured due to the fire effect and the out of balance force $\Delta \mathrm{F}$ is evaluated (Equation 11).

$$
\Delta F\left(t_{1}\right)=F_{P}\left(t_{1}\right)+F_{N}\left(t_{1}\right)=-K_{P} \alpha L_{P} T\left(t_{1}\right)
$$

f. To restore the equilibrium, the incremental displacement $\Delta u$ will be calculated using Equation 12 . In the calculation process, the stiffness of the PS and the NS are accounted for. This is the main difference with the first generation method (in which only the stiffness of the NS was considered) and the most important contribution that ensures the stability of the method.

$$
\Delta u\left(t_{1}\right)=-\left(K_{N}+K_{P}{ }^{*}\right)^{-1} \Delta F\left(t_{1}\right)=\frac{K_{P}}{K_{N}+K_{P}{ }^{*}} \alpha L_{P} T\left(t_{1}\right)
$$

Note that $K_{P} *$ is the stiffness of the PS considered in the calculations (the estimation of the real stiffness) while $K_{P}$ is the real stiffness of the PS measured in the furnace. Since the real stiffness of the PS is unknown during the HFT, a constant value can be considered in the calculations (for example, the initial tangent stiffness of the PS). This simplification will imply additional iterations in order to satisfy the objectives of the HFT and this aspect will be discussed later on.

For the studied 1 DoF linear system, the stiffness considered in the calculations $K_{P} *$ is equal to the real stiffness $K_{P}$ of the PS (based on the hypothesis that the stiffness of the PS remains constant during this exercise).

g. The new calculated displacements (Equation 13) are imposed on the PS and NS. The time needed to perform these calculations and to adjust the new displacements in the furnace $\left(\Delta t_{P}\right)$ is accounted for. Thus, the displacement computed at the time step $t_{1}$ is applied on the PS at the time $t_{1}+\Delta t_{P}$.

$$
u\left(t_{1}\right)=u\left(t_{0}\right)+\Delta u\left(t_{1}\right)=\frac{K_{P}}{K_{N}+K_{P}{ }^{*}} \alpha L_{P} T\left(t_{1}\right)
$$

h. The new imposed displacements will generate new reaction forces in the PS and NS. For the next time step $t_{2}$, the reaction force of the PS is given by Equation 14 and the reaction force of the NS is computed using Equation 15. 


$$
\begin{aligned}
& F_{P}\left(t_{2}\right)=-K_{P} \alpha L_{P}\left(T\left(t_{2}\right)-\frac{K_{P}}{K_{N}+K_{P}^{*}} T\left(t_{1}\right)\right) \\
& F_{N}\left(t_{2}\right)=\frac{K_{N} K_{P}}{K_{N}+K_{P}{ }^{*}} \alpha L_{P} T\left(t_{1}\right)
\end{aligned}
$$

i. The incremental displacement $\Delta u\left(t_{2}\right)$ is computed based on the out of balance force $\Delta F\left(t_{2}\right)$. This yields to a value of displacement $u\left(t_{2}\right)$ expressed by Equation 16 .

$$
u\left(t_{2}\right)=u\left(t_{1}\right)+\Delta u\left(t_{2}\right)=\frac{K_{P}}{K_{N}+K_{P}{ }^{*}} \alpha L_{P} T\left(t_{2}\right)
$$

Expanding the above equations for $n$ time steps, the reaction force of the PS can be expressed by Equation 17, while the reaction force of the NS is computed using the Equation 18. Therefore, the computed displacement for $n$ time steps to be imposed at the interface of the substructures can be calculated using Equation 19.

$$
\begin{aligned}
& F_{P}\left(t_{n}\right)=-K_{P} \alpha L_{P}\left(T\left(t_{n}\right)-\frac{K_{P}}{K_{N}+K_{P}{ }^{*}} T\left(t_{n-1}\right)\right) \\
& F_{N}\left(t_{n}\right)=\frac{K_{N} K_{P}}{K_{N}+K_{P}{ }^{*}} \alpha L_{P} T\left(t_{n-1}\right) \\
& u\left(t_{n}\right)=\frac{K_{P}}{K_{N}+K_{P}{ }^{*}} \alpha L_{P} T\left(t_{n}\right)
\end{aligned}
$$

In the next sections, the new method will be analyzed in order to observe whether all the objectives of HFT are fulfilled and, if so, under which conditions.

\subsection{Stability}

Equations 6-9 presented in the Section 3 show that the stability of the HFT when considering the first generation method depends on the stiffness ratio $R$. No conditionality on the stiffness ratio $R$ is observed in Equations 17-19 of the new method.

\subsection{Equilibrium and compatibility}

The equilibrium condition implies equal absolute values of interface forces, while the compatibility condition implies equal displacements at the interface of the substructures. The compatibility is ensured since equal displacements are imposed on the interface of the substructures every time step.

The equilibrium must be satisfied when the computed displacement is imposed at the interface, i.e. $t_{n}+\Delta t_{P}$, therefore, the out of balance force $\Delta F\left(t_{n}+\Delta t_{P}\right)$ expressed by Equation 20 must be equal to zero.

$$
\Delta F\left(t_{n}+\Delta t_{P}\right)=F_{P}\left(t_{n}+\Delta t_{P}\right)+F_{N}\left(t_{n}+\Delta t_{P}\right)=-K_{P} \alpha L_{P}\left(T\left(t_{n}+\Delta t_{P}\right)-\frac{K_{N}+K_{P}}{K_{N}+K_{P}^{*}} T\left(t_{n}\right)\right)
$$

The equilibrium is satisfied if the term in the parenthesis (Equation 20) is equal to zero. If the delay time $\Delta t_{P}$ is small enough (tends to $0 \mathrm{~s}$ ), then the temperature $T\left(t_{n}+\Delta t_{P}\right)$ is approximately equal to the temperature $T\left(t_{n}\right)$. If the stiffness matrix $K_{P} *$ used in the calculations equals the real 
stiffness matrix of the PS $K_{P}$, then the out of balance force tends to a value of zero and the equilibrium is satisfied.

If the temperature $T\left(t_{n}+\Delta t_{P}\right)$ is different than the temperature $T\left(t_{n}\right)$ (assuming that $\left.K_{P}{ }^{*}=K_{P}\right)$, then the out of balance force is different than zero and the equilibrium is not satisfied. This can occur when $\Delta t_{P}$ is large enough and leads to the increase of temperature in the PS from $t_{n}$ to $t_{n}+\Delta t_{P}$.

In the case of the $1 \mathrm{DoF}$ linear elastic system, the stiffness of the PS remains constant during the HFT $\left(K_{P}^{*}=K_{P}\right)$. Therefore, equilibrium is achieved when the delay time $\Delta t_{P}$ does not lead to an increase of the temperature in the heated substructure.

In a real HFT, as the stiffness of the PS is generally unknown (the initial tangent stiffness may be considered in the calculations), several iterations would normally be needed at each time step to converge to the correct solution. Yet, in a fire test, the evolution of temperatures in the PS cannot be put on hold for the period requested to perform the iterations at every time step. The temperatures keep on increasing during the time needed to perform the calculations in the computer and for the testing equipment to apply the corrections of displacements, and this continuously modifies the stiffness of the PS, the restraint forces, etc. Hence, the convergence process is aiming at a state of equilibrium that is constantly changing. As a result, it is not relevant to distinguish between iterations and time steps. Instead, the test can be performed by applying continuously Equation 19 with a cycling frequency which leads to the satisfaction of all the conditions of HFT, i.e. stability, equilibrium, compatibility and the reproduction of the correct solution (Sauca et al., 2016b).

\subsection{Reproduction of the correct solution}

For the linear 1 DoF system presented in Figure 2, the "correct" displacement of the node 2 is given by Equation 21. No exterior forces are acting on the system and only the PS is exposed to fire, where $E_{P}$ is the Young modulus of the PS and $A_{P}$ is the sectional area of the PS.

$$
u\left(t_{n}\right)=\frac{E_{P} A_{P}}{K_{N}+K_{P}} \alpha T\left(t_{n}\right)
$$

The displacement generated by the novel method of HFT (Equation 19) is equal to the correct solution (Equation 21) when the stiffness of the PS considered in the calculations $K_{P} *$ is equal to the real stiffness of the PS $K_{P}$.

Since continuous iteration process is employed during the HFT, the generated solution will converge to the correct solution. Nevertheless, for the real hybrid fire tests, the time step and the estimation of the stiffness of the PS must be selected with the aim to satisfy all the objectives of the HFT. A parametric analysis is recommended for the proper selection of these parameters before the start of the test.

In conclusions, the novel method is stable, able to ensure the interface equilibrium and compatibility, and to reproduce the correct solution at the interface for a proper selection of some parameters.

\section{HFT APPLIED TO A CASE STUDY}

A 2D reinforced concrete moment resisting frame is analyzed by means of hybrid fire testing in a virtual environment (both substructures are modelled numerically). In the performed case study, the behavior of the NS is predefined by using a predetermined matrix while the response of the PS is modeled in SAFIR ${ }^{\circledR}$. It must be understood that although a full geometrically and materially non-linear analysis is performed in SAFIR ${ }^{\circledR}$ to mimic what the behavior of a real specimen would be in a real furnace, this information is not used in the algorithm of the hybrid test, where the stiffness of the PS is kept constant during the test.

Figure 3 presents the moment resisting concrete frame and the beam above the fire compartment is the only structural element exposed to ISO fire, therefore representing the PS. The rest of the structure is the NS which remains cold during the analysis. 


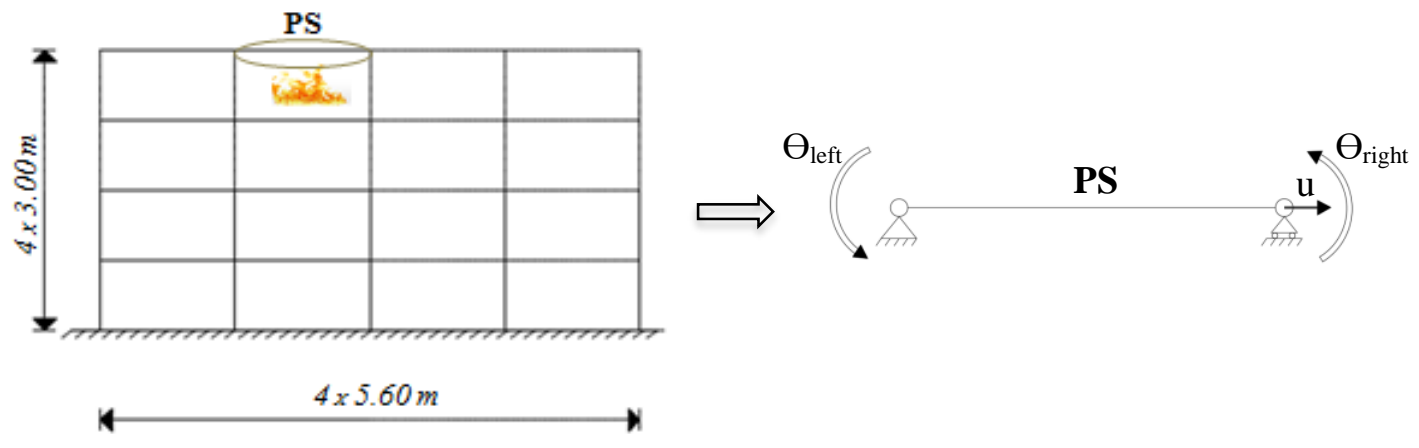

Figure 3. PS subtracted from the moment resisting concrete frame

Local degrees-of-freedom are controlled during the HFT and the selection is done based on the capability of the furnace, in this example the horizontal displacement $u$ and the rotations on the supports $\theta_{\text {left }}$ and $\theta_{\text {right }}$. The horizontal displacement induces the axial force $H$ and the rotations on the supports, the bending moments $M_{\text {left }}$ and $M_{\text {right }}$. The behavior of the NS can be represented by using a predetermined matrix (defined before the HFT) as is the case in this example, or a finite element model. The advantage of the predetermined matrix is that the time calculation of the NS is virtually zero, whereas by using the finite element model, the time calculation can be significant. The condensation from 6 global degrees of freedom to the 3 local degrees of freedom is captured in the predetermined matrix of NS (Sauca, 2016). At ambient temperature, the stiffness ratio for the horizontal DoF is equal to 0.20 , respectively 2.53 for the rotation on the left support and 2.48 for the rotation on the right support. With the first generation method, in order to avoid instability, a force control procedure is requested for the axial DoF and a displacement control procedure for the rotational DoFs, at least at ambient temperature.

An automatic procedure was developed in SAFIR in order to perform virtual hybrid fire tests (Sauca, 2016) when the behavior of the NS is represented using a predetermined matrix. The HFT subroutine computes the interface displacements to be imposed at the interface of the substructures at every time step.

Before the hybrid fire test, the stiffness of the PS must be estimated. For this example, the stiffness of the PS is considered constant, with a value equal to 1.50 times the initial tangent stiffness. Parametric analysis is done to determine the proper value of the time step in order to fulfill the objectives of the hybrid fire testing, i.e. stability, compatibility, equilibrium and the reproduction of the correct solution.

Figure 5 illustrates the interface forces versus displacement/rotations for different values of the time step, i.e. $\mathrm{t}=1,10,30$ and $60 \mathrm{~s}$. The correct solution expresses the results of the analysis performed on the complete structure (no substructuring).

Two cases are presented, one when the NS is considered to have a linear elastic behavior (first column graphs) versus the case when the NS develops material nonlinearities (second column graphs). In the virtual HFT, in both cases, the NS is defined by a constant predefined matrix.

It can be observed that no instability occurs; the first objective of HFT is thus fulfilled.

Equilibrium and compatibility between the PS and NS are ensured if the solution of one substructure matches the solution of the other substructure. For this specific example, compatibility and equilibrium are satisfied only with a time step of $1 \mathrm{~s}$. For larger time steps, the solution of the PS diverges from the solution of the NS; equilibrium and compatibility are not ensured. This example illustrates that the choice of the time step is crucial for accurate results. It can be shown that the value chosen for the stiffness of the PS considered in the calculations influences the maximum value of the time step that ensures accurate results. If the real stiffness of the PS is overestimated in the calculations, then the number of iterations required to reach the equilibrium and compatibility is increasing, therefore the selected time step should decrease. If the stiffness of the PS is well estimated during the test, then the required number of iterations is reduced compared with the previous situation, therefore the value of the selected time step is higher. If the stiffness of the 
PS is underestimated, then the instability can occur during the test (Sauca, 2017). Therefore, before the start of the hybrid fire test, it is crucial first to define the value of the stiffness of the PS to be used in the calculation process, and then to determine the value of the time step which leads to accurate results, so the objectives of the HFT are satisfied. Thus, the objective is to approximate the real stiffness of the PS (the underestimation should be avoided) so that the number of iteration required is not too high and at the same time, the instability is not induced.
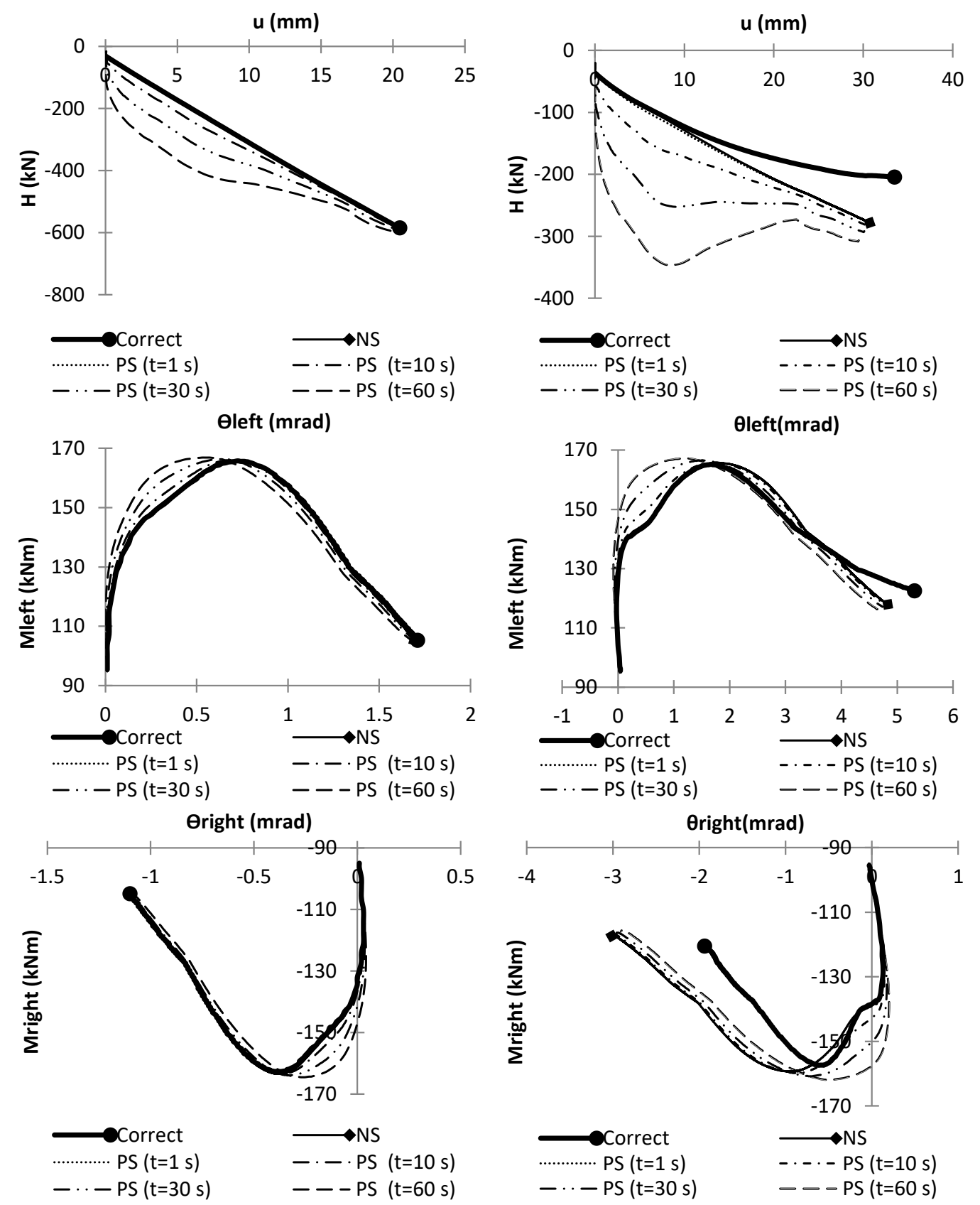

a) Linear NS

b) Nonlinear NS

Figure 5. The interface force/moments versus interface displacement/rotations for the axial and rotational degrees-of-freedom when a) Linear NS and b) Nonlinear NS

Beside stability, equilibrium and compatibility, the solution of HFT must reproduce the correct solution. For this specific example, the correct solution is well reproduced for the case when the NS is considered to have a linear elastic behavior (in Figure 5 (a), the plots for Correct, NS and 
PS ( $t=1 \mathrm{~s})$ lie on top of each other). For the case when the NS is nonlinear, the correct solution is well reproduced, except toward the end of the test. A slight divergence from the correct solution is observed and this is due to the fact that a constant predetermined matrix is used to represent the behavior of the NS. Even if the NS is cold during the entire HFT, nonlinearities develop in this concrete structure towards the end of the test. It has been checked that the time when the solution given by the HFT diverges from the correct solution coincides with the time when the nonlinearities develop in the correct solution. One way to avoid this divergence is to use variable predetermined matrices (the nonlinear solution is approximated by linear segments) or to model the NS in a finite element model.

\section{CONCLUSIONS}

Hybrid fire testing HFT is a promising technique to test individual structural elements while at the same time considering the effect of the surrounding structure. The methodology considered in the former HFT is conditionally stable on the stiffness ratio between the NS and PS since only the stiffness of the NS is considered in the calculation process of the interface forces or displacements. A new method is proposed in this paper, where the dependency on the stiffness ratio is eliminated by introducing the stiffness of the PS in the calculation process. The new method is stable, but at the same time ensures equilibrium and compatibility at the interface of the substructures and moreover, is able to reproduce the correct solution. In the first part of the paper, the first generation method and the new method are analyzed analytically considering as an example a 1 degree-of-freedom (DoF) linear system. After that, a concrete moment resisting frame has been analyzed in the virtual environment by means of HFT. The PS is a concrete beam, while the remainder represents the NS. Three DoFs are controlled at the interface, and it has been observed that all the objectives of HFT are fulfilled. Moreover, a parametric study is performed in order to observe the influence of the time step on the results. The results show that the selection of the time step influences the accuracy of the results.

\section{REFERENCES}

Franssen, J.-M. (2005), "SAFIR, A Thermal/Structural Program Modelling Structures under Fire", A.I.S.C. Engineering Journal, 42 (3) 143-158.

Korzen, M., Ziener, K.-U. and Riemen, S. (2002), "Some Remarks on the Substructuring Method Applied to Fire Resistance Tests of Columns", World Congress on Housing, Housing Construction-An Interdisciplinary Task, 9-13 September 2002, Coimbra, Portugal.

Mostafaei, H. (2013a), "Hybrid fire testing for assessing performance of structures in fire -Methodology", Fire Safety Journal, 58, 170-179.

Mostafaei, H. (2013b), "Hybrid fire testing for assessing performance of structures in fire -Application", Fire Safety Journal, 56, 30-38.

Robert, F., Rimlinger S. and Collignon, C. (2010), "Structure fire resistance: a joint approach between modelling and full scale testing (substructuring system)", 3rd fib International Congress.

Saouma, V.E., and Sivaselvan, M.V. (2008), Hybrid simulation: Theory, implementation and application, Taylor and Francis, London, UK.

Sauca, A., Gernay, T., Robert, F., Tondini, N. and Franssen, J.M. (2016a), Stability in Hybrid Fire Testing, 9th International Conference on Structures in Fire, 2016, June 8-10, pp.836-843.

Sauca, A., Gernay, T., Robert, F., Tondini, N. and Franssen, J.M. (2016b), "A novel method for Hybrid Fire Testing", 6th European Conference on Structural Control, 2016, July 11-13.

Sauca, A. (2016), Development and implementation of a methodology for hybrid fire testing applied to concrete structures with elastic boundary conditions, $\mathrm{Ph}$. D. thesis, University of Liege, Liege, Belgium.

Schulthess, P., Neuenschwander, M., Knoblock, M. and Fontana, M. (2016), "Consolidated Fire Analysis - Coupled Thermo-mechanical Modelling for Global Structural Fire Analysis", 9th International conference on Structures in Fire, 8-10 June, pg. 819-826.

Tondini, N., Abbiati G., Possidente L. and Stojadinovic B. (2016), "A Static Partitioned Solver for Hybrid Fire Testing", $9^{\text {th }}$ International Conference on Structures in Fire, June 8-10, pg. 819-826.

Whyte, C.A., Mackie, K.R. and Stojadinovici, B. (2016), "Hybrid Simulation of Thermomechanical Structural Response”, Journal of Structural Engineering, 142(2): 04015107-1 - 04015107-11. 